\title{
Chapter 3 \\ Size Matters in Primate Societies: \\ How Social Mobility Relates to Social \\ Stability in Tibetan and Japanese Macaques
}

\author{
Lixing Sun, Dong-Po Xia, and Jin-Hua Li
}

\subsection{Introduction}

Social mobility refers to vertical movement of positional status of members in a society. In human societies, it refers to a wide range of upward or downward change in metrics such as income, social stature, education, and others that show some level of stratification (Lipset and Bendix 1992). As a focal issue in equality, social justice, economic development, and social stability in human societies (e.g., Wilkinson and Pickett 2009; Breen 2010; Cox 2012; Matthys 2012; Corak 2013; Clark 2014; Piketty 2014), social mobility has drawn a broad and sustained interest from social thinkers, social activists, and concerned citizens and is among the most important current research topics in social science disciplines including economics, sociology, and political science. Recently, interest in social mobility has also been on the rise in biological, psychological, and medical sciences. This is largely due to the discoveries that, along with other effects, social status can affect hormones (especially those

\footnotetext{
L. Sun $(\bowtie)$

Department of Biological Sciences, Primate Behavior and Ecology Program, Central Washington University, Ellensburg, WA, USA

e-mail: Lixing@cwu.edu

D.-P. Xia

School of Life Sciences, Anhui University, Hefei, China

International Collaborative Research Center for Huangshan Biodiversity and Tibetan Macaque Behavioral Ecology, Anhui, China

J.-H. Li

School of Resources and Environmental Engineering, Anhui University, Hefei, Anhui, China International Collaborative Research Center for Huangshan Biodiversity and Tibetan Macaque Behavioral Ecology, Anhui, China

School of Life Sciences, Hefei Normal University, Hefei, Anhui, China e-mail: jhli@ahu.edu.cn
} 
related to stress such as glucocorticoids), immunity, and health in humans and nonhuman primates (e.g., Marmot et al. 1991; Sapolsky 2005; Seabrook and Avison 2012; Snyder-Mackler et al. 2016). As such, social mobility is among the key factors determining the well-being of primates and other social animals.

The level of social mobility is critically important for a society, especially from the long-term perspective. Both low and high social mobility are believed to weaken and disrupt social stability. A persistently low mobility will necessarily deprive low-ranking members of opportunities for social advancement, which in turn may increase the probability of revolts and revolutions. An extremely high mobility, on the other hand, will make a society constantly in flux. Therefore, a stable society is a dynamic one with the level of mobility bounded within a certain range, beyond which society may become chaotic or even collapse. Such a system dynamics view thereorized for human societies has gained some popularity recently (Acemoglu et al. 2018). New conception aside, empirical tests are hard to carry out for the obvious reason that human societies are usually too complex in structure and too diverse in culture to pursue such studies. In this respect, testing mobility-related hypotheses is more feasible in nonhuman primates because their societies are usually smaller in size and simpler in structure than human societies, including most huntergatherer societies (Price and Brown 1985; Hamilton et al. 2007). Furthermore, understanding how social mobility influences primate societies is interesting and important in its own right because it can give us a new tool to investigate the dynamics and evolution of primate societies.

(Note that social primates can live in groups of varying complexity in social structure, ranging from simple linear hierarchy in some macaque species (Macaca spp.), multilevel societies such as those in geladas (Theropithecus gelada) and snubnosed monkeys (Rhinopithecus spp.), to highly organized human communities governed by formal or informal norms and laws. Because social mobility is a feature of an operationally definable social unit or organization regardless of the level of its organizational complexity, we here use the word "society" synonymously with "social group" for both human and nonhuman primates for the purpose of finding general and comparable patterns in social mobility.)

There are two types of social mobilities: intragenerational and intergenerational. Intragenerational mobility refers to upward and downward movement of social stature for members in a society within their lifetimes. For instance, in a Tibetan macaque society, each member has a 3-to-1 ratio of upward versus downward mobility in a year (Sun et al. 2017). Because status change tends to accumulate over time, intragenerational mobility is often measured per time unit, such as a month, a year, or a decade, to be comparable across studies. For this, it is more accurately known as the rate of social mobility (Clark 2014). Intergenerational mobility, on the other hand, refers to the status change between two consecutive generations. In American society, for instance, income mobility declined from 92\% for people born in 1940 to 50\% for people born in 1985 (Chetty et al. 2017). In this chapter, we will focus on intragenerational mobility.

Social mobility can be absolute or relative. In social sciences, absolute mobility refers to the total number of individuals, whereas relative mobility refers to the 
probability of an individual (or percentage of all individuals) moving from one social stratum to another. A society can be high in absolute mobility but low in relative mobility or vice versa. The key behind this paradox lies in the size of a society. For example, in terms of absolute mobility, two members moving up one step in rank in a group of 30 are twice as mobile as one member for the same rank change in a group of 15 during the same period of time, but the two groups are equally mobile in relative mobility, both $6.67 \%$ for this period of time. As this hypothetical example illustrates, absolute mobility does not consider the size of a society. Thus, relative mobility can better reflect the overall condition for a society and is most commonly used in characterizing upward or downward mobility (Heckman and Mosso 2014; Simandan 2018). Accordingly, social mobility, unless specified, refers to relative mobility hereafter.

The difference between absolute and relative mobility indicates that society size can play an important role in social mobility. Unfortunately, few attempts have been made to address this issue due to the reason that most mobility research has been conducted on large human societies in industrial nations. As such, society size is usually not of great interest because it has no perceivable effect on social mobility. In nonhuman primate societies, however, group size can be magnitudes smaller, typically a few dozen (up to a few hundred in some species such as baboons and snub-nosed monkeys). A group of a few dozen, such as in Tibetan macaques, M. thibetana (Li 1999), can be vastly different in social organization from a group of a few hundred, such as in Sichuan snub-nosed monkeys, $R$. roxellana (Zhang et al. 2006) or Japanese macaques, $M$. fuscata (Mori et al. 1989). Therefore, to understand how group size affects mobility, we compared two macaque species, the Tibetan macaque and the Japanese macaque, in this study.

Before we present our results, we briefly review some key issues related to the study of social mobility.

\subsection{Social Mobility and Opportunity}

In the study of animal social behavior, proximate benefits and costs for a set of alternative behaviors (such as mating versus feeding or fighting) are often estimated in terms of time and energy. However, although researchers are keenly aware of the benefit and cost in terms of opportunity intrinsic to every behavioral decision (e.g., when an animal hides in a den, it loses the opportunity to feed or mate), quantitative measurement has not been attempted due to practical difficulties in empirical studies. Measuring social mobility may provide just such a way to gauge gain or loss in opportunity for social advancement for social animals such as primates. Because mobility is an aggregate measure for a society, it can provide a baseline (the average or the expected) for opportunity in social advancement for all members in a social group (Sun et al. 2017). With this baseline as a benchmark, the opportunity 
consequences of many behaviors, biological identities, and social relationships of individuals (such as dispersal, conflict, cooperation, kinship, age, and sex) can be quantitatively assessed in terms of gains or losses in social opportunity relative to this benchmark. In other words, these behaviors, identities, or relationships can alter an individual's expected upward and downward mobility; that is, the benchmark. For instance, a monkey deciding to transfer from Group A to Group B must accept a complete loss in opportunity for social advancement in Group A, in exchange for a gain in opportunity in Group B. Whether this dispersal event is adaptive depends on the difference between the two opportunity measurements.

\subsection{Social Mobility and Social Stability}

In his classic, Democracy in America, the French political scientist Alexis de Tocqueville first noticed that mobility has a profound effect on the stability of human societies (de Tocqueville 1835/2002). Social scientists have since developed several explanations for why mobility is essential for stability (e.g., Lipset and Bendix 1992; Erikson and Goldthorpe 1994; Chetty et al. 2017). Studies in developed nations show that high mobility is closely related to equality and stability, and low mobility engenders inequality, which may cause a spectrum of social problems detrimental to society in general and democracy in particular (e.g., Wilkinson and Pickett 2009). It is a consensus now that a certain level of mobility is essential for the persistence of a society in the long run. Such a broadly held belief, however, hinges on the veracity of the hypothesis that mobility leads to stability. Unfortunately, this hypothesis, though logically compelling, is still short of empirical evidence (Bai and Jia 2016).

In reality, the relationship between mobility and stability is profound. On the one hand, an absolutely stable society without mobility can deprive its low-ranking members of opportunities to advance their social status, resulting ultimately in a loss in their fitness relative to that of high-ranking members (Sun 2013; Sun et al. 2017). As such, low-ranking members in a stagnant society will have a strong incentive to resort to revolt against the existing order so as to gain critical resources (such as food and mates) that are otherwise inaccessible for them. Therefore, an extremely unequal society with little mobility is evolutionarily unstable (Sun 2013). It tends to periodically experience major disruptions in the form of rebellion and revolution in humans (Bai and Jia 2016). It is in this sense that a certain level of mobility is essential for long-term social stability (Sun et al. 2017). On the other hand, mobility is the opposite of stability. That is, a higher level of mobility will necessarily decrease stability. This implies that too much mobility may also destabilize a society, which, beyond a certain point, may cascade into social upheaval (e.g., Clark 2014). However, this hypothesis, despite a broad theoretical interest, remains untested as well. 


\subsection{Measuring Social Mobility in Primate Societies}

As mentioned earlier (Sect. 3.1), intragenerational mobility is normally calculated as the rate of status change in society within a specified time period (e.g., Lammam et al. 2012; Clark 2014). Technically, it can be measured either as the mean value in the rate of rank change or using a linear regression analysis between the ranks of all individuals in two consecutive time periods, $t$ and $t+1$. For the latter method, the rate of social mobility is thus $1-b$, where $b$ is the slope of the regression line ranging from 0 to 1 (Clark 2014; Sun et al. 2017). If $b$ is 0 , there is no mobility and, in our case, no rank change between two consecutive years for all adults involved. If $b$ is 1 , there is no stability. In primates, social mobility has been measured only in Tibetan macaques, where the rate of rank mobility was calculated for all adults on a yearly basis (Sun et al. 2017).

(Note that this linear regression method belongs to a statistical analysis known as time series analysis. Although time series analysis is relatively unfamiliar to researchers in biological sciences, it is commonly used in economic and business analysis such as GDP growth from year to year and stock performance from quarter to quarter. By emphasizing changes over time, the influence of policies or the efficacy of management regimes behind these changes can be statistically analyzed. As we can see, one distinct strength of time series analysis in addressing behavioral, ecological, and evolutionary issues lies in that it allows researchers to statistically track changes over time, which in turn may lead to the unveiling of the underlying factors that have significant influences on the changes. As such, time series regression is a standard method in the study of social mobility in social sciences.)

To complicate the issue, however, dominance rank can be measured as absolute rank or relative rank. Absolute rank refers to the raw rank data regardless of group size. Relative rank, however, is standardized according to group size. (Note: absolute and relative rank is a way of measuring dominance rank for all members in a group. They have no relationship to, and should not be mistaken as, absolute and relative mobility introduced in Sect. 3.1.) As we have already discussed, the distinction is not meaningful (or even feasible to measure) when a society is very large and the hierarchy is highly nonlinear, such as an industrial society, which tends to show complex forms of strata. However, the distinction becomes important when a society is made of only a few dozen individuals typical of many macaques species. For example, we can see a huge difference between the fifth ranked individual in a group of ten versus the 25th ranked individual in a group of 50 when measured in absolute rank. However, when measured in relative rank, these two individuals are ranked the same, both in the middle of the hierarchies. Clearly, measurement of social mobility will be affected by whether we use absolute or relative rank. Yet, little is known as to how using relative or absolute rank will affect mobility measurements.

In this study, we probed into the critical issue of how group size affects intragenerational mobility by using both absolute and relative rank in Tibetan and Japanese macaques. For Tibetan macaques, data about the hierarchical relationships among all adult females come from our 29-year consecutive observation on one 
group (YA1) at Mt. Huangshan, Anhui, China. Details about the dataset and how the data were collected are available in Sun et al. (2017). For the Japanese macaque, we obtained data from a published paper, where 11 years of hierarchical relationships among adult females were documented in a group on Koshima Island from 1957 to 1986 (Mori et al. 1989).

Age is well known to affect dominant status in primates (e.g., de Vries 1998; Packer et al. 2000; Alberts et al. 2003; Bayly et al. 2006; Balasubramaniam et al. 2013). Thus, comparing individuals of the same age class is critically important. However, because male and female Tibetan macaques sexually mature at different ages (seven for males and five for females, see Li 1999), we only included adult females that were 7 years or older in our analysis for two reasons. First, it allowed us to compare male and female adults of the same age class in our discussion here and in other places (Sun et al. 2017). Second, the ranks of immature females from the same mother are inversed. That is, the later born are higher in dominance rank than their elder sisters (Li 1999). Therefore, the inclusion of the 5- and 6-year-old females may introduce an unnecessary confounding factor, which may in turn reduce the accuracy in measuring social mobility for adults. The data about rank and rank change in Japanese macaques were collected from adult females of 5 years or older (Mori et al. 1989). Although the research in the Japanese macaque spanned 29 consecutive years, only 11 years of rank data were published.

For the study groups of both species, we used the original rank data for all adult females as their absolute ranks. We then calculated the relative ranks by scaling all absolute rank data into values between 0 (alpha female) and 1 (omega female) using a simple conversion formula:

$$
R R=(A R-1) /(n-1)
$$

where $R R$ and $A R$ refer to relative and absolute rank, respectively, and $n$ is the size of the group. We then measured mobility using the same time series regression method as that detailed in Sun et al. (2017); that is, by using linear regression between rank $R$ $(t)$ in year $t$ and rank $R(t+1)$ in year $t+1$ to measure the annual rate of mobility for all adult group members (see Clark 2014) for both Tibetan and Japanese macaques. (Note that sample sizes about yearly rank changes were calculated as individualyears, rather than individuals in most statistics used in behavioral studies. In other words, each independent data point or sampling unit here is individual-year. This is a standard practice in time series analysis.) In our study, none of the datasets used for measuring mobility violated the normality assumption required for linear regression based on Kolmogorov-Smirnov test. As we have discussed in an earlier section, social mobility can be used to quantify the availability of social opportunities (e.g., Breen 2010; Clark 2014; Sun et al. 2017), which then can be used to quantify the benefit and cost of a behavior in terms of gain or loss in opportunity for social advancement. Here, we calculated net social mobility (subtracting the downward mobility from the upward mobility) and then used it to compare the difference between adult females of the two species. For the ease of reading, the statistical 
methods used are provided as they occur in the next section. As usual, all tests were two-tailed with 0.05 as the a priori level of significance for any statistical difference.

\subsection{Results: Social Mobility in Tibetan and Japanese Macaques}

From the adult female perspective (which may also be true if all individuals are included, if the number of adults, subadults, and infants is scaled in roughly similar proportions), the group size of Japanese macaques (33.09 \pm 7.968SD; range, 21-49; $n=11)$ was more than three times larger than that of Tibetan macaques (7.62 $\pm 2.624 \mathrm{SD}$; range, 3-12; $n=29$; Mann-Whitney U-test, $U=385$; $n_{1}=11 ; n_{2}=29 ; p=0.000$; see Fig. 3.1).

Using absolute rank, we found that group members experienced a larger rate of rank change in Japanese macaques than in Tibetan macaques. This was adequately reflected in both the mean $\left(U=40,821, n_{1}=192, n_{2}=204, p=0.013\right)$ and variation (Levene test, $L=65.07, d f_{1}=1, d f_{2}=394, p=0.000$, Fig. 3.2) of annual rank change for Japanese macaques. Also, Japanese macaques had a higher rate in both upward (Chi-square test with Yates' correction, $\chi^{2}=13.187, d f=1, p<0.001$ ) and downward movement $\left(\chi^{2}=18.705, d f=1, p=0.000\right)$ in hierarchy and a lower probability of staying in the same rank $\left(\chi^{2}=37.436, d f=1, p=0.000\right)$ than Tibetan macaques (Fig. 3.3). However, when we compared mobility between the two species using the regression method (rate of mobility, 0.115 for Japanese macaques and 0.144 for Tibetan macaques), the difference was not statistically significant ( $t$-test for the slope, $t=0.658, d f=392, p=0.510$, Fig. 3.4).

For Tibetan macaques, mobility was higher using relative rank than using absolute rank, although the difference was marginally insignificant $(t=1.706, d f=380$, $p=0.089$ ). For Japanese macaques, the situation was the opposite. That is, mobility

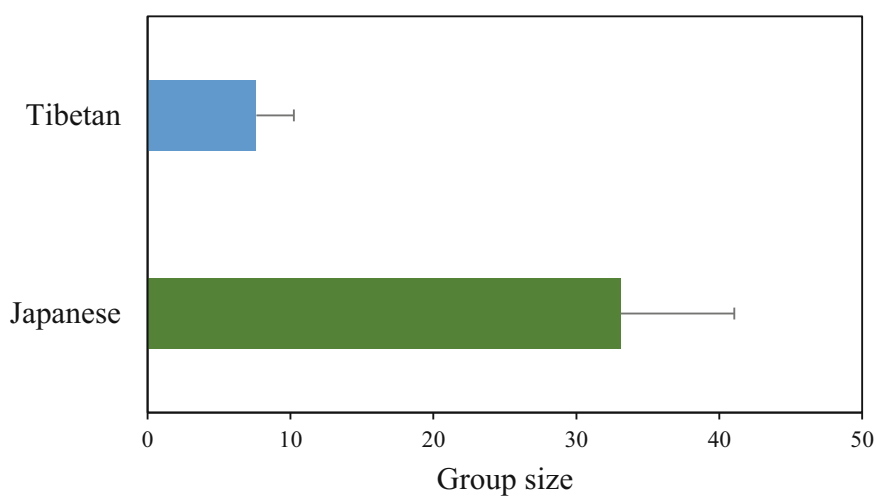

Fig. 3.1 Comparison of the mean group size (only adult females) in Tibetan and Japanese macaques. Error bars are standard deviations 


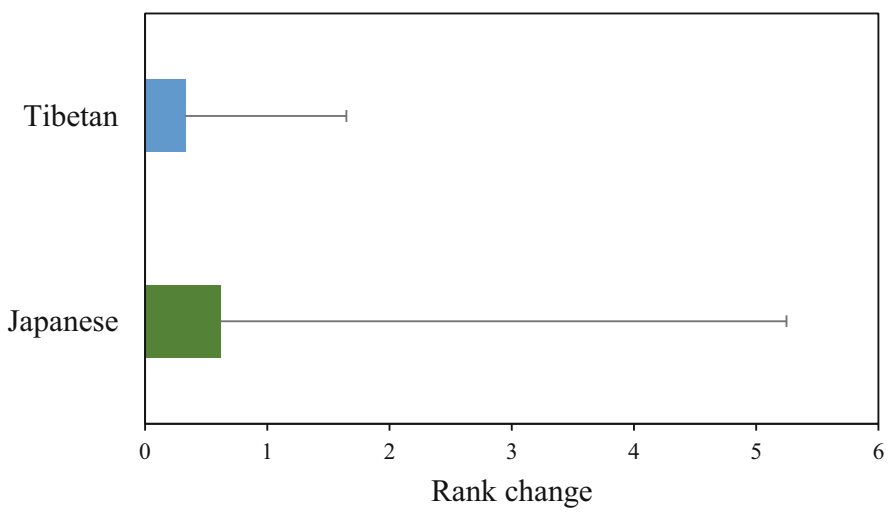

Fig. 3.2 Mean annual rate of absolute rank change in Tibetan and Japanese macaques. Error bars are standard deviations

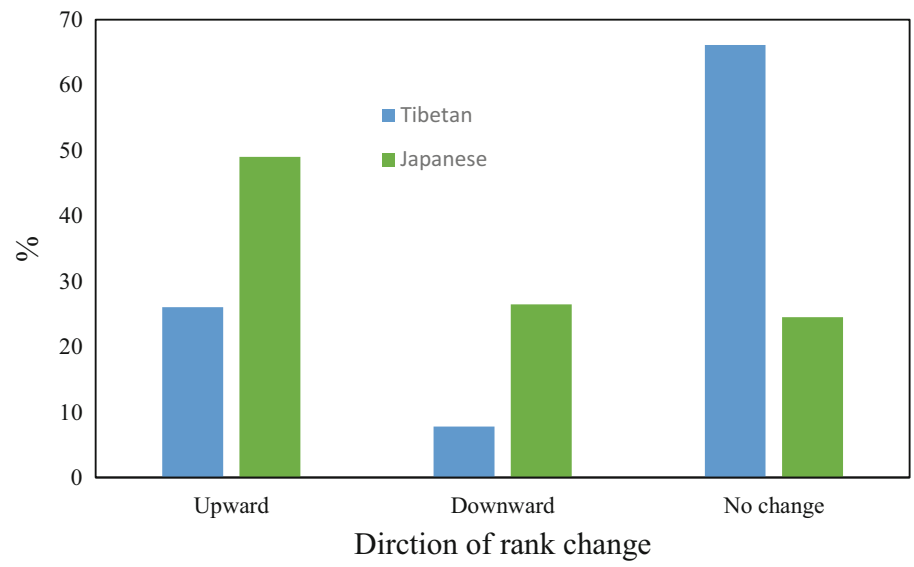

Fig. 3.3 Comparison of the frequency (\%) of annual rank change (upward, downward, and no change) between Tibetan and Japanese macaques

was significantly lower when relative rank was used than when absolute rank was used $(t=2.291, d f=404, p=0.037)$. Between-species comparison shows that there was no significant difference in absolute rank mobility, but a highly significant difference in relative rank mobility $(t=3.901, d f=392, p=0.0001$; see Fig. 3.5).

\subsection{Discussion}

This chapter presents a case study of two macaque species to illustrate how social mobility can be used as a new approach to the study of social dynamics as well as an in-depth analysis to explore the effect of group size. Our results show that social 
a. Tibetan macaque (absolute)

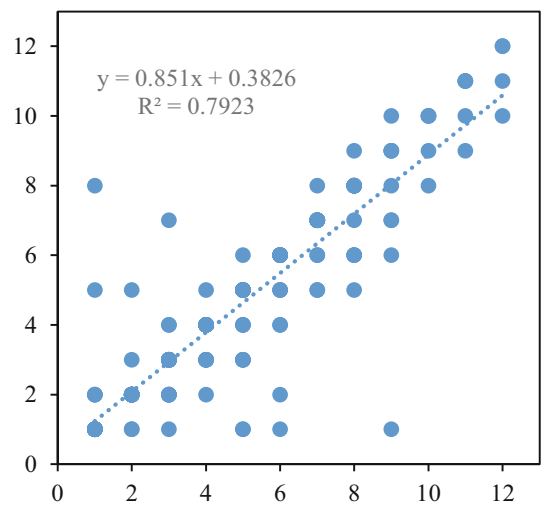

c. Tibetan macaques (relative)

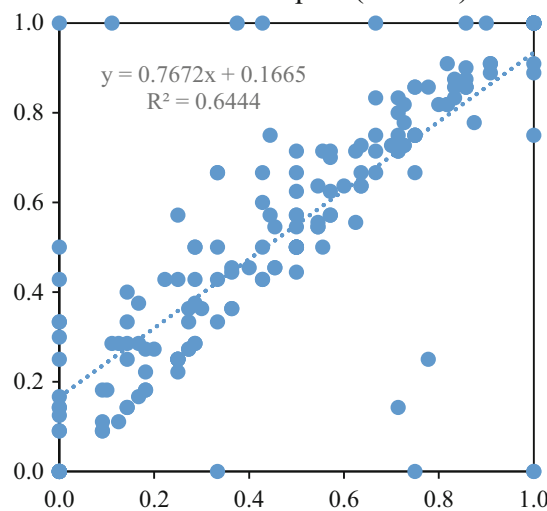

b. Japanese macaque (absolute)

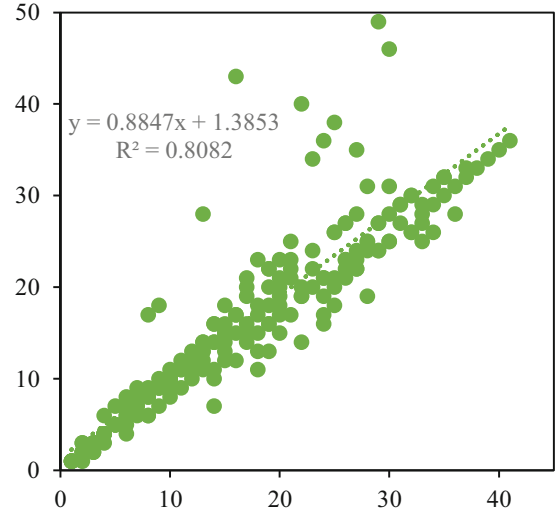

d. Japanese macaque (relative)

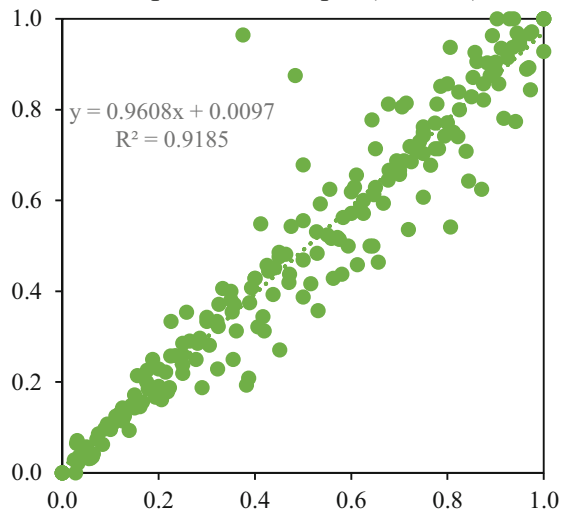

Fig. 3.4 Social mobility as measured by absolute ("absolute") and relative ("relative") rank in Tibetan and Japanese macaques using time series regression analysis. The $\mathrm{X}$-axis indicates rank in year $t$, and the Y-axis indicates rank in the next year, year $t+1$, for all adult individuals used in the analysis. Note that fewer data points than sample size shown in Panel (a) is due to data overlap

mobility can unveil new and unexpected insights into the social structure of primate societies. Our analyses show that, although Japanese macaques appeared to be more dynamic in rank changes, as shown by the higher mean and larger variation than Tibetan macaques, social mobility as measured in absolute rank was virtually the same for the two species. This result was surprising considering that Tibetan macaques and Japanese macaques are two different species with marked difference in so many aspects of their behavior and social organization (see Thierry 2011), despite some shared sociological traits such as matrilineality in social organization and strong despotism (see below).

One factor we particularly examined was group size, which was much larger in Japanese macaques than in Tibetan macaques. (Note that this difference may be 


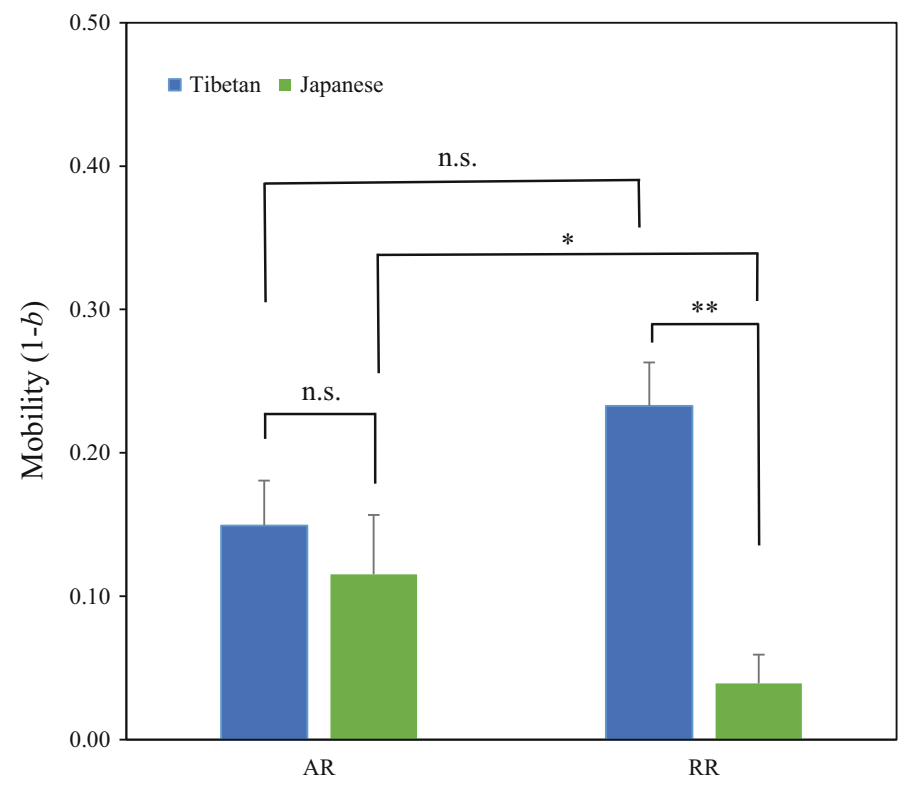

Fig. 3.5 Within- and between-species comparisons of social mobility using absolute (“AR”) and relative ("RR") rank for the Tibetan and Japanese macaque. "n.s." stands for not significant, "asterisk" indicates $p<0.05$, and "double asterisk" represents $p<0.001$

slightly less prominent had only females of 7 years or older been included in the analysis for Japanese macaques.) This apparent discrepancy between the results from measuring the rate of rank change and from time series regression analysis might be due to two reasons. One is that, although the Japanese macaque troop studied had a larger rate of rank change, much of the significance was offset by a higher level of variation (Fig. 3.2). Also, using a nonparametric test for a non-normal dataset (Mann-Whitney U test here) might also lead to a slightly different statistical result from a parametric linear regression analysis.

Comparing the two species, we found that Tibetan macaque society appeared much more stable than that of the Japanese macaque in absolute rank change, despite no difference in mobility between the two species. Indeed, Tibetan macaques live in a small, simple, matrilineally structured group of strictly linear hierarchy (Li 1999; Berman et al. 2008) and their dominance hierarchy usually remains stable for a prolonged period of time, at least several months (Li 1999). (These behavioral conditions are particularly favorable for observing and determining social status for every adult in the hierarchy with little ambiguity. That is why Tibetan macaques are a highly desirable model species in the evolutionary study of behavior, a point compellingly addressed in the previous two chapters). Even so, social mobility in Tibetan macaques turned out to be similar to that in Japanese macaque. It appears that a larger group (society) may require a higher level of rank change to maintain the same level of mobility as a smaller group (society). Conversely, with the same level of mobility, a larger group would be more dynamic in terms of rank change. From 
the dynamic stability point of view, therefore, a larger society may require more rank change than a smaller society to maintain its stability in the long run, if the amount of mobility for long-term social stability is assumed to be approximately the same for all societies.

Another major reason for why the two species had similar levels of mobility may lie in the fact that they both form highly despotic societies; Grade 1 (highly despotic) for the Japanese macaque and Grade 2 (despotic) for the Tibetan macaque (Thierry 2000, 2011; Berman et al. 2004, 2006). Apparently, the two species we analyzed here may not be different enough in dominance style to show marked difference in social mobility. In other words, social mobility is likely to be related to dominance style. One logical hypothesis is that despotism may suppress social mobility. If true, this hypothesis would predict that a more relaxed or egalitarian primate society should have a higher level of mobility. This, however, requires a broader comparison with a larger diversity in dominance style, ideally in the genus Macaca, which has been well studied for the topic (Thierry 2011).

The most surprising finding in our study was that social mobility could vary a great deal depending on whether absolute or relative rank was used. In Tibetan macaques, the group size of adult females could be so small that rank data, once standardized to be within the range between 0 and 1, appear erratic (see in Fig 3.4c). Since relative rank can change with group size, even though there is no change in absolute rank, small changes can become prominent, which may in turn lead to increment in mobility. Obviously, the smaller the group is, the higher the mobility will become when relative rank is used. In Japanese macaques, on the contrary, larger rank changes (with the extreme records of 9 ranks upward and 27 ranks downward in a year in Japanese macaques compared with up 8 and down 7 in Tibetan macaques) became smaller after these changes were scaled to be within the range between 0 and 1 . Clearly, this effect increases with group size. As a result, mobility decreases with increasing group size. These changes led to the counterintuitive result that, despite Japanese macaques appearing to be more dynamic in terms of absolute rank change, their society actually had a lower level of mobility than Tibetan macaques when relative rank was used.

This part of our results leads to an unexpected insight into social evolution. That is, group size may have a major impact on group dynamics. In both macaque species we examined, the net mobility was positive for social advancement. This may be true for most primate societies, in which social rank is more or less related to seniority and/or tenure. Consequently, as higher-ranking individuals become senile or die, younger, lower-ranking individuals can move up over time. So, even without major disruptive events, net mobility tends to be positive (upward). Overall, if everything else is equal, individuals in general should prefer smaller groups to larger groups, not only because absolute ranks go up (as the length of hierarchy becomes shorter) for most members but also because mobility goes up as well in terms of relative rank. Therefore, individuals in a larger society may have more incentive to break off to form smaller societies. This interesting and intriguing difference in mobility when measured in absolute versus relative rank may provide a novel explanation for why group fission is so common whereas group fusion is so rare in primates. It also sheds new light on the balkanization of human societies, happening in so many nations 
under the name of regional autonomy and sovereignty rather than the other way around. Although it is too early to claim that such events in human and nonhuman primate societies share biological roots, their uncanny similarities should warrant a close and serious examination on such possibilities from the evolutionary perspective.

Acknowledgments We are grateful to Mike Huffman, Peter Kappeler, and an anonymous reviewer for their helpful comments and constructive suggestions, which led to a considerable improvement in the clarity of our chapter. We also thank the Huangshan Garden Forest Bureau for their permission and support of this work and H.B. Cheng's family for their outstanding logistic support to our study. Rose Amrhein proofread the chapter. This work was supported in part by grants from the National Natural Science Foundation of China (No. 31772475; 31672307).

\section{References}

Acemoglu D, Egorov G, Sonin K (2018) Social mobility and stability of democracy: re-evaluating de Tocqueville. Q J Econ 133:1041-1105

Alberts SC, Watts HE, Altmann J (2003) Queuing and queue jumping: long term patterns of dominance rank and mating success in male savannah baboons. Anim Behav 65:821-840

Bai Y, Jia R (2016) Elite recruitment and political stability: the impact of the abolition of China's civil service exam system. Econometrica 84:677-733

Balasubramaniam KN, Berman CM, De Marco A et al (2013) Consistency of dominance rank order: a comparison of David's scores with I\&SI and Bayesian methods in macaques. Am J Primatol 75:959-971

Bayly KL, Evans CS, Taylor A (2006) Measuring social structure: a comparison of eight dominance indices. Behav Processes 73:1-12

Berman CM, Ionica CS, Li J (2004) Dominance style among Macaca thibetana on Mt. Huangshan, China. Int J Primatol 25:1283-1312

Berman CM, Ionica CS, Dorner M et al (2006) Postconflict affiliation between former opponents in Macaca thibetana on Mt. Huangshan, China. Int J Primatol 27:827-854

Berman CM, Ogawa H, Ionica CS (2008) Variation in kin bias over time in a group of Tibetan macaques at Huangshan, China: contest competition, time constraints or risk response? Behaviour 145:863-896

Breen R (2010) Social mobility and equality of opportunity Geary lecture spring 2010. Econ Soc Rev 41:413-428

Chetty R, Grusky D, Hell M et al (2017) The fading American dream: trends in absolute income mobility since 1940 . Science $356: 398-406$

Clark G (2014) The son also rises: surnames and the history of social mobility. Princeton University Press, Princeton NJ

Corak M (2013) Income inequality, equality of opportunity, and intergenerational mobility. J Econ Perspect 27:79-102

Cox MW (2012) Myths of rich and poor: why we're better off than we think. Basic Books, New York

De Tocqueville A (2002) Democracy in America. Regnery Publishing, Washington, DC

De Vries H (1998) Finding a dominance order most consistent with linear hierarchy: a new procedure and review. Anim Behav 55:827-843

Erikson R, Goldthorpe JH (1994) The constant flux: a study of class mobility in industrial societies. Clarendon Press, Oxford

Hamilton MJ, Milne BT, Walker RS et al (2007) The complex structure of hunter-gatherer social networks. Proc Biol Sci 274:2195-2202 
Heckman JJ, Mosso S (2014) The economics of human development and social mobility. Annu Rev Econ 6:689-733

Lammam C, Karabegović A, Veldhuis N (2012) Measuring income mobility in Canada. Fraser Institute, Social Science Electronic Press, Vancouver, BC

Li J (1999) The Tibetan macaque society: a field study. Anhui University Press, Hefei

Lipset SM, Bendix R (1992) Social mobility in industrial society. Transaction Publishers, Brunswick

Marmot MG, Smith GD, Stansfeld S et al (1991) Health inequalities among British civil servants: the Whitehall II study. Lancet 337:1387-1393

Matthys M (2012) Cultural capital, identity, and social mobility. Routledge, New York

Mori A, Watanabe K, Yamaguchi N (1989) Longitudinal changes of dominance rank among the females of the Koshima group of Japanese monkeys. Primates 30:147-173

Packer C, Collins DA, Eberly LE (2000) Problems with primate sex ratios. Philos Trans R Soc Lond B Biol Sci 355:1627-1635

Piketty T (2014) Capital in the 21st century. Belknap, Cambridge, MA

Price TD, Brown JA (1985) Aspects of hunter-gatherer complexity. In: Price TD, Brown JA (eds) Prehistoric hunter-gatherers: the emergence of cultural complexity. Academic, Orlando, FL, pp 3-20

Sapolsky RM (2005) The influence of social hierarchy on primate health. Science 308:648-652

Seabrook JA, Avison WR (2012) Socioeconomic status and cumulative disadvantage processes across the life course: implications for health outcomes. Can Rev Sociol 49:50-68

Simandan D (2018) Rethinking the health consequences of social class and social mobility. Soc Sci Med 200:258-261

Snyder-Mackler N, Sanz J, Kohn JN et al (2016) Social status alters immune regulation and response to infection. Science 354:1041-1045

Sun L (2013) The fairness instinct: the Robin Hood mentality and our biological nature. Prometheus Books, Amherst, NY

Sun L et al (2017) The prospect of rising in rank is key to long-term stability in Tibetan macaque society. Sci Rep 7(1):7082

Thierry B (2000) Covariation of conflict management patterns across macaque species. In: Aureli F, de Waal FBM (eds) Natural conflict resolution. University of California Press, Berkeley, CA, pp $827-843$

Thierry B (2011) The macaques: a double-layered social organization. In: Campbell CJ, Fuentes A, McKinnon KC et al (eds) Primates in perspective. Oxford University Press, New York, pp 229-241

Wilkinson R, Pickett K (2009) The spirit level: why greater equality makes societies stronger. Bloomsbury Press, New York

Zhang P, Wanatabe K, Li B et al (2006) Social organization of Sichuan snub-nosed monkeys (Rhinopithecus roxellana) in the Qinling Mountains, Central China. Primates 47:374-382

Open Access This chapter is licensed under the terms of the Creative Commons Attribution 4.0 International License (http://creativecommons.org/licenses/by/4.0/), which permits use, sharing, adaptation, distribution and reproduction in any medium or format, as long as you give appropriate credit to the original author(s) and the source, provide a link to the Creative Commons licence and indicate if changes were made.

The images or other third party material in this chapter are included in the chapter's Creative Commons licence, unless indicated otherwise in a credit line to the material. If material is not included in the chapter's Creative Commons licence and your intended use is not permitted by statutory regulation or exceeds the permitted use, you will need to obtain permission directly from the copyright holder.

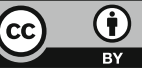

